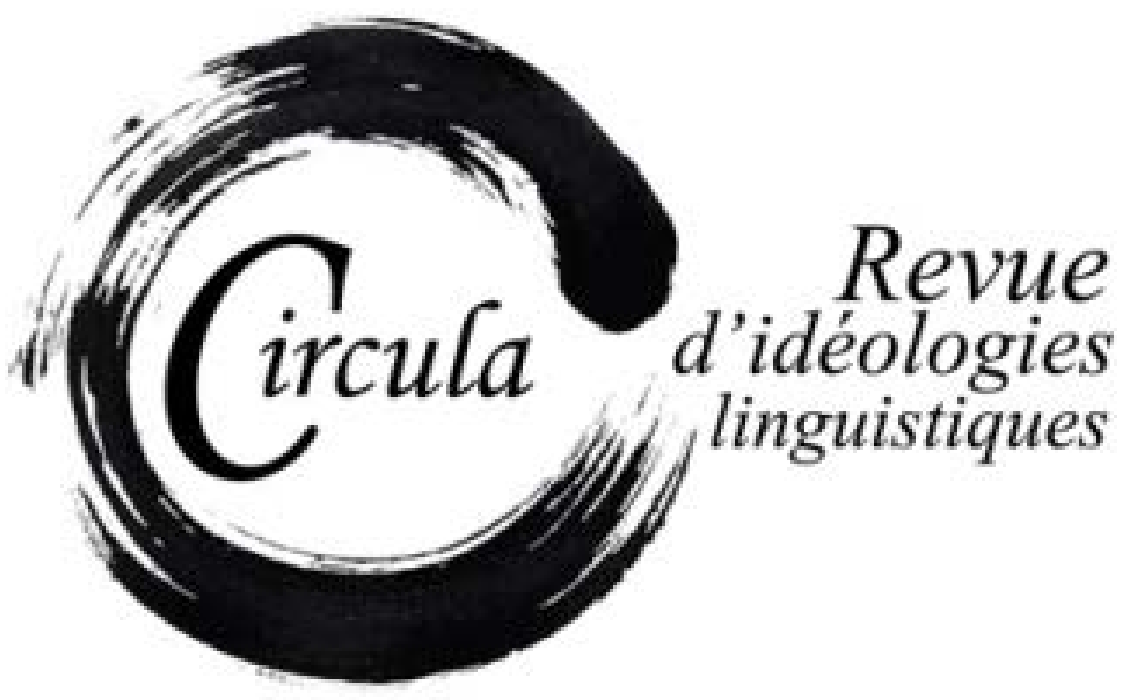

TITRE: LES INTERNAUTES FONT LA POLICE : PURISME LANGAGIER ET SURVEILLANCE DU DISCOURS D'INFORMATION EN CONTEXTE NUMÉRIQUE

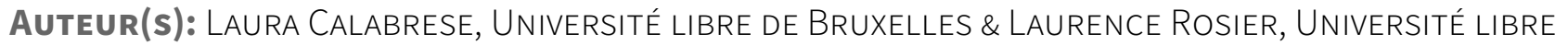
DE BRUXELLES

ReVUe: CiRCulA, NUMÉRO 2, PAGES 120-137

ISSN: 2369-6761

DiRecteurs: Wim Remysen et SABINE SchWARZE

URI: HTTP://HDL.HANDLE.NET/11143/7980

DOI: $10.17118 / 11143 / 7980$ 


\title{
Les internautes font la police : purisme langagier et surveillance du discours d'information en contexte numérique
}

\author{
Laura Calabrese, Université libre de Bruxelles \\ Icalabre@ulb.ac.be
}

Laurence Rosier, Université libre de Bruxelles

Irosier@ulb.ac.be

Résumé : Cet article examine la manière dont le discours des lecteurs de journaux en ligne contribue à forger un discours normatif sur la langue des journalistes. Nous analysons un corpus de commentaires dans lesquels les lecteurs critiquent des articles journalistiques en se focalisant sur les erreurs de grammaire ou de langue. Nous essayons d’identifier les éléments qui déclenchent le débat, ainsi que les arguments qui servent à l'arrêter. L'objectif est de montrer que la norme n'est plus détenue uniquement par les producteurs du discours journalistique mais partagée avec les lecteurs, qui jouent un rôle de plus en plus grand dans la surveillance et la correction du discours médiatique.

Mots-clés : discours des lecteurs ; commentaires en ligne; norme ; discours prescriptif

Abstract: This paper addresses the issue of Internet-based readers' discourse, and how it contributes to build a prescriptive discourse on journalists' written language. A corpus of comments where the readers criticize news items by spotting grammar or language mistakes will be analyzed. The elements which sparkle the debate will be identified, as well as which arguments are used to put an end to it. The aim is to show that the norm is no longer on the producers' side, but shared with the readers, who play an increasing role in watching and sanctioning media discourse.

Keywords: readers' discourse; online comments; norm; prescriptive discourse 


\section{Introduction}

Dans « l'eldorado » que constitue l'Internet comme « nouveau corpus » (Calabrese, 2011), il est une forme d'interactivité et de co-construction discursive qui concerne particulièrement la manière dont les imaginaires et les représentations sont façonnés: le commentaire des internautes. Poster un commentaire est une intervention discursive, plus ou moins brève, sous une forme plus ou moins argumentée et contrainte (notamment sous la surveillance d'un modérateur et selon la nétiquette) sur un énoncé antérieur. Les commentaires font partie intégrante des pratiques renouvelées par le numérique; sur la Toile (Facebook, sites d'information, forums, sites de vente), ils semblent la forme d'intervention la plus courante et occupent une place centrale dans les discours sociaux, ce qui souligne leur caractère performatif.

Une brève typologie des fonctions du commentaire met en avant les aspects suivants :

- la valeur laudative,

- la valeur critique à polémique,

- la valeur méliorative,

- la valeur ludique,

- la valeur d'attestation de la crédibilité,

- $\quad$ la mise en avant de son point de vue (Meunier et Rosier, 2014).

Ces fonctions occupent des places diverses dans les interactions en ligne et donnent naissance à des sous-conversations entre les internautes commentateurs, qui pourront être dominées par certaines fonctions (par exemple un fil conducteur laudatif ou polémique). L'alternance et/ou la combinatoire des fonctions donnent lieu à des échanges très complexes du point de vue interactionnel. On peut repérer des profils d'internaute suivant leur façon de commenter et de privilégier des interventions (par exemple, celui qui ne poste que des commentaires à caractère ludique sera vu comme le « comique de service » ou le bon esprit).

Par ces interventions, les internautes contribuent, entre autres, à la reformulation des discours médiatiques, en faisant évoluer les pratiques de lecture et d'écriture contemporaines, notamment de la presse (Calabrese, 2014c). L'avènement des dispositifs participatifs a effectivement une incidence sur le discours d'information, car à côté de celui-ci a pris place un discours des lecteurs qui ne peut plus être qualifié de « populaire » ou de « profane », tant il est traversé par le discours savant et/ou expert que les publics médiatiques produisent (Calabrese, 2014a). Une des caractéristiques de ce discours est de faire circuler des représentations normatives et prescriptives sur la presse (Calabrese, 2014b), qui rejoignent le discours normatif sur la langue déjà observé ailleurs (Rosier, 2008). Nous savons effectivement à quel point les internautes sont devenus des vecteurs importants dans la propagation 
et la circulation d'un « discours normatif » sur la langue (Osthus, 2004 ; Paveau et Rosier, 2008) ; il nous reste à montrer quel est leur rapport avec l'écriture journalistique, qui apparaît souvent dans les discours épilinguistiques des non-linguistes comme « dégradée » (Canut, 2007).

Le corpus des commentaires permet d'observer la dynamique entre le purisme des internautes-lecteurs et la pratique des journalistes, qui disposent d'un imaginaire linguistique propre (voir l'article d'Antoine Jacquet dans le présent volume). Même si le public intervenant représente un petit pourcentage de la totalité des lecteurs, et même si tous les lecteurs n'ont pas la possibilité d'intervenir², les réactions observables permettent de mettre au jour une certaine dynamique des publics avec le discours d'information, devenu à l'heure actuelle un discours extrêmement surveillé.

Dans cet article, nous nous pencherons spécifiquement sur les commentaires métalangagiers des lecteurs de la presse d'information généraliste. Si les commentaires, en règle générale, sont l'expression de représentations sociales, ceux-ci abordent la question de la norme linguistique sous l'angle de la correction grammaticale dont les journalistes devraient être les garants. Ces commentaires s'appuient donc sur un imaginaire linguistique dans lequel certains locuteurs/scripteurs occupent des postures sociales privilégiées de propagation et de pratique de la bonne norme. Dès lors, la langue des journalistes se trouve régulièrement incriminée lorsque des fautes sont identifiées dans des publications sur la Toile. Mais les commentaires nous révèlent également ce que le public estime avoir comme rôle dans cet espace public interactif, que ce soit surveiller la langue ou, au contraire, relâcher la surveillance en raison de l'environnement numérique ou en vertu d'une posture antipuriste. Comme nous le verrons, c'est là que la possibilité d'un contre-discours normatif va s'énoncer.

Plus particulièrement nous examinerons la manière dont, à partir d'une information en ligne ne traitant pas d'un fait de langue ou de grammaire, la conversation par commentaire prend soudain un cours non seulement linguistique mais grammatical, voire puriste (ou antipuriste). Il s'agira de repérer les « démarreurs » discursifs de ce type d'intervention et d'analyser ensuite la manière dont les échanges se poursuivent, entre prescription, proscription et sanction.

Le corpus ici présenté a été collecté au cours de plusieurs mois dans des journaux de référence en ligne. Nous avons réuni une trentaine d'articles provenant des journaux Le Monde, Libération, Le Figaro et Le Soir, ainsi que quelques exemples tirés du réseau social Facebook. Au cours de la collecte, nous avons décidé d'incorporer des billets des blogues Big Browser et Des risques et des hommes du Monde ( 6 sur tout le corpus), dont les billets présentaient une concentration élevée d'énoncés métalinguistiques dans la section des commentaires. Le premier blogue est tenu par la rédaction du monde.fr, alors que le deuxième est un blogue invité tenu par un expert. Cette particularité du

1. Osthus (2004) montre par exemple que les discussions sur les forums dédiés à la langue produisent et reproduisent un discours émotionnel et normatif sur la langue.

2. Dans plusieurs journaux, il faut être abonné pour intervenir. Il y a également des restrictions d'espace, celles dictées par la nétiquette ainsi que par les modérateurs des commentaires. On peut également mentionner des restrictions liées à l'univers d'attente des lecteurs-internautes, plus difficiles à saisir car elles se construisent dans l'interaction. 
corpus nous a conduit à étendre notre réflexion à la question de l'environnement discursif, après avoir constaté que la dynamique du blogue favorisait des échanges d'une nature particulière qu'on n'observait pas sur le site du journal, et notamment la production de commentaires métadiscursifs. Pour faciliter la visualisation des échanges entres les internautes, nous présentons chaque énoncé accompagné d'une lettre.

\section{Le commentaire puriste}

Nous définissons le purisme comme une activité métalinguistique à tendance prescriptive et/ou proscriptive. Ses modalités varient en fonction des genres de discours et des postures (bienveillante ou violente) ; de façon générale, le purisme s'ancre dans un imaginaire linguistique fondé sur le bon usage et une vision «classiste » de la langue. Le purisme est donc une forme d'attitude sociolinguistique stigmatisante qu'Internet a contribué à propager. En effet, la Toile permet à la norme de se dire et de se redire sous des formes inédites, car elle permet à de nouveaux genres d'exister et dans ce sens elle renouvelle la langue et rend poreuses les frontières entre le discours d'expert et le discours non expert. Enfin, la Toile permet à différents registres et aux différentes orthographes de se côtoyer, alors que dans la plupart des espaces sociaux ils sont hiérarchisés. Comme le note Ruth Amossy, «les forums de discussion de la presse électronique donnent aux individus la possibilité de "rencontrer" les opposants avec lesquels ils pourraient, sans cela, n'avoir aucune possibilité de débattre » (2011: 38). Dès lors, la confrontation des pratiques et des normes est inévitable. Le commentaire sur la Toile est pour cette raison un terreau d'expression privilégié des attitudes puristes.

Dialogique par excellence, le commentaire devient également dialogal, ou plutôt polylogal, dans la section que les journaux en ligne lui réservent, dont la dynamique est proche de celle du forum de discussion. Le dispositif des commentaires s'inscrit plus largement dans « l'écrit d'écran » (l'expression est d'Emmanuel Souchier [1996]), lequel favorise le regard « micro » (y compris sur les éléments de langue) et suscite d'office une réaction du lecteur (Calabrese, 2014a, 2014c), notamment des interventions puristes des lecteurs les plus lettrés. Si faire des fautes semble menacer le dispositif communicationnel, qu'il s'agit donc de préserver, pour certains le relevé et la mention des erreurs menacent également le lien social, puisque la pratique devient discriminante. Ainsi, des internautes seraient disqualifiés d'une discussion uniquement pour une formulation maladroite ou fautive (image 1). 


\begin{tabular}{|c|c|}
\hline & 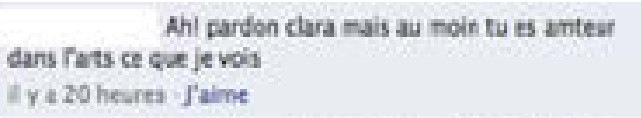 \\
\hline & 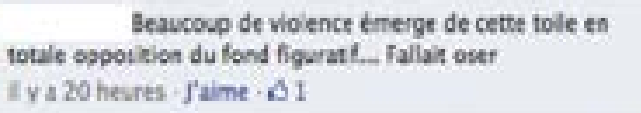 \\
\hline & $\begin{array}{l}\text { Lest tout ce que u trouver a cire au tant cue } \\
\text { Visiteur quili nalivete' Monsieur Bruno } \\
\text { i ya } 19 \text { heures f'alme }\end{array}$ \\
\hline & 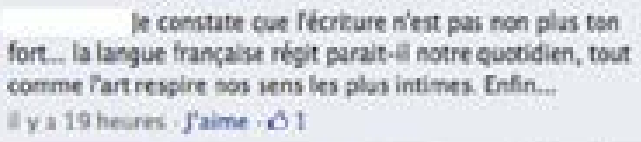 \\
\hline & $\begin{array}{l}\text { est universel je pense que to es inteligent, Noliere if } \\
\text { if y } 18 \text { hesres fraime }\end{array}$ \\
\hline & 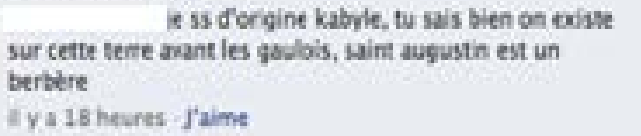 \\
\hline 6 & 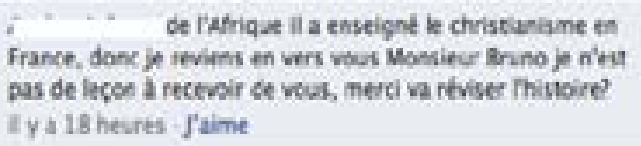 \\
\hline$\Rightarrow$ & $\begin{array}{l}\text { TArts il nest pas a vous aussi vou dectiez } \\
\text { reviser bien mistoire } \\
\text { ly a } 18 \text { hesres - J'aime }\end{array}$ \\
\hline & 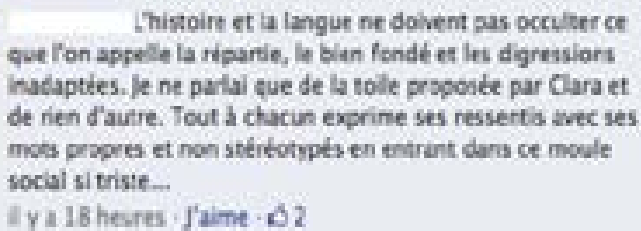 \\
\hline
\end{tabular}

\section{Image 1}

Sur la Toile, le purisme s'exprime sans forcément faire référence à un univers de classe, mais plutôt à un arrière-plan culturel partagé (image 2), même s'il reste rattaché à un monde lettré, comme on le voit dans l'image 3.

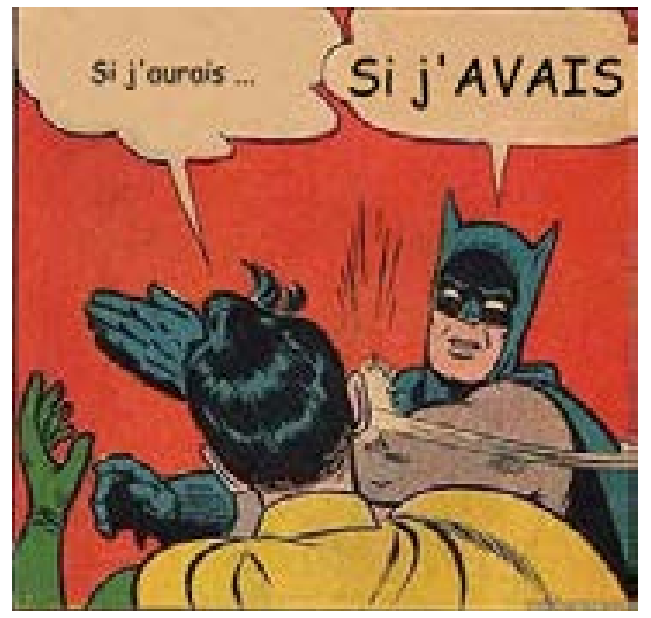

Image 2 
9 octobre was mobile av

"C'est gratifiant de se dire que dans l'université dans laquelle je fais partie..." (Bon, on ne parle pas du aNobel de littérature non plus)

Je n'aime plus - Commenter - Partager

\section{Image 3}

L'observation des interventions sur le Net permet de définir des profils prototypiques d'internautes selon leur mode d'intervention sur la langue. Ces profils ont fait notamment l'objet d'une approche sociologique "spontanée ", souvent réalisée par les participants mêmes aux différentes formes d'intervention sur la Toile. On citera le petit ouvrage de Mat Hild, Et toi, t'es qui ? Petite typologie des profils facebook, paru en 2012, qui répertorie notamment le rigolo, le compulsif du statut ou le poète. Nous pouvons également mentionner la figure du troll, à laquelle les commentateurs font souvent allusion, qui peut être vu comme un stoppeur des échanges sur Internet. Pour ce qui concerne la langue et ses normes, on retiendra particulièrement le populaire grammar nazi (image 4), remarqueur moderne, défini comme un membre généralement investi de responsabilités, qui insiste pour préserver l'intégrité de la langue. Il est généralement très sévère envers ceux qui bafouent les règles (même les plus avancées) de la grammaire et de l'orthographe. Cette figure existe indépendamment de la langue dont il est question.

\section{WHEN COMFORTING A GRAMMAR NAZI}

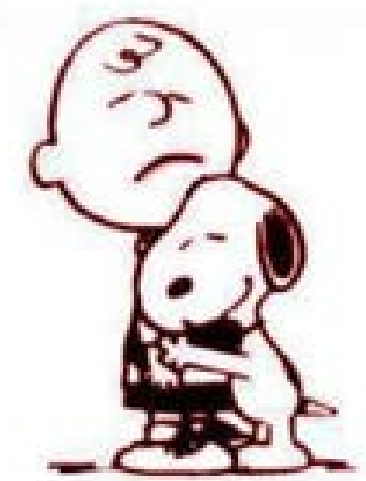

\section{ALWAYS SAY SOFTLY, "THERE, THERR, THEY'RE"}


Image 4 
Le commentaire sur leWeb acquiert des caractéristiques spécifiques selon l'environnement discursif. Dans l'espace virtuel des journaux en ligne, le rapport entre les internautes, celui qu'ils entretiennent avec le journal et les journalistes, la manière dont ils imaginent leur rôle face au discours d'information et le rôle de la presse dans la société déterminent la pratique du commentaire, qui émerge dans un dispositif sociodiscursif contraint. Le commentaire est encadré par le dispositif sociotechnique même, qui présente une série de restrictions matérielles et des objets de discours prédéfinis, même si, comme nous le verrons, il est facile de s'en détourner. Le dispositif met en rapport deux paires de co-énonciateurs : internaute-internaute, internaute-journaliste ou expert. Ces rapports discursifs ne sont pas équilibrés, les journalistes ayant une présence faible dans la section des commentaires et gardant par ailleurs le contrôle du dispositif. Le dispositif met également en rapport des types de discours, à savoir le discours d'expert, le discours « professionnel » des journalistes (qui n'est pas à proprement parler un discours d'expert) et le discours non professionnel des lecteurs. Le réseau discursif qui s'établit ainsi n'est pas le même que l'on retrouve dans les forums ou des blogues autres que ceux incorporés au journal.

Le rapport entre lecteurs et journalistes crée une tension entre les gatekeepers, ceux qui produisent l'information, et ceux qui la consomment mais de manière active, comme des produsers (Bruns, 2010), et à qui on donne la possibilité de corriger, d'amender et de compléter l'information. Cette tension est visible dans le brouillage des positionnements énonciatifs, qui nous conduit à nous demander quel est le détenteur de la norme et qui est habilité à adopter une position experte en matière de langue. Le type d'énoncés que nous analysons dans cet article, c'est-à-dire ceux qui portent sur la langue des journalistes (mais aussi des autres internautes), est un observatoire privilégié de ce phénomène.

\section{La pratique puriste et ses modalités d'intervention}

Examinons maintenant notre corpus, dans lequel les internautes pointent une faute et font ainsi dévier les commentaires (voire les initient) vers un échange purement langagier. Concrètement comment se présentent leurs interventions? L'enjeu est le repérage immédiat, avant toute discussion sur l'objet initial de l'article pour établir le cadre normatif de la discussion. L'internaute puriste ne peut pas s'empêcher de repérer les fautes sur un mode parfois humoristique (1a), bienveillant (1c), souvent violent (2a):

(1) Furosémide Teva : l'agence du médicament a-t-elle surréagi ? [titre] (http://securitesanitaire.blog.lemonde.fr 30.6.2013)

(1a) « surréagit » ???? Petite pensée pour une de mes profs de prépa qui aurait déchiré la page devant toute la classe... «On ne badine pas avec la grammaire messieurs dames ! »

(1b) Nul doute que j'aurais eu une mauvaise note au bac! J'ai corrigé, merci [réponse du journaliste] 
(1c) Bonjour, Un commentaire qu'il n'est pas indispensable de publier : il y a une faute d'accord dans le titre (pas de «t» au participe passé final). Bien à vous.

(1d) Mea culpa, j'ai manqué de vigilance avec mon correcteur. Merci du signalement, j'ai corrigé [réponse du journaliste]

(2) USA : une victime de viol se bat pour que le coupable ne puisse pas voir leur enfant [titre] (lefigaro.fr 24.9.2013)

(2a) demande le droit de visite «SUR » l'enfant???? Vraient???? Mais où etes- vous donc allé à l'école??? quelle langue française vous a-t-on appris????

(2b) Je ne sais pas ce qui m’horrifie le plus: le drame vécu par cette mère ou le nombre de fautes contenues dans l'article...

Ces exemples disqualifient de façon générale un texte en lui assénant le verdict sans appel : « plein de fautes », « truffé de fautes »... (2b supra, 3 infra), le « trop » étant la manifestation d'une défaillance scripturale inacceptable. L'exaspération se traduit par la surabondance de la modalité exclamative (1a et 2a).

(3) Une affiche montrant Cristina Kirchner avec le pape fait polémique en Argentine [titre] (lemonde.fr 02.08.2013)

S’il-vous-plaît... Relisez-vous, ne publiez pas sans faire relire par un correcteur, je ne sais pas, faites quelque chose, mais ne laissez pas publier de telles fautes...

D’autres épinglent des erreurs ponctuelles relevant pêle-mêle de la concordance des temps, de l'emploi des prépositions, de l'accord du participe passé, des néologismes, bref des zones de turbulence de la norme : en effet, les puristes se cristallisent sur des cas où les règles ne sont pas rigoureuses et où il y a justement une certaine variation, ce qu'ils ne supportent pas. Une fois l'erreur commise, ils renvoient aux figures et aux outils de la norme, tels que la figure du professeur et les ouvrages normatifs classiques. La première est convoquée à plusieurs reprises (1a), de même que l'exemple a contrario, ci-dessous, dans lequel c'est l'enseignant lui-même qui, ayant commis des fautes, se voit stigmatisé (4b) :

(4) 10/20 au lieu de 12/20 pour réussir, des étudiants dénoncent [titre] (lalibre.be 11.11.2013)

Le décret Marcourt a déjà fait couler beaucoup d'encre [texte]

(4a) Mais quand comprendra-t-on que les cotations ne sont en aucun cas « objectives »?

Je me souviens que toutes les cotes obtenues par les jeunes enfants au cour de danse oscillaient entre $90 \%$ et $99 \%$.......

Lorsqu'il attribue une cote, le correcteur pense « réussite ou non », « très bonne réponse ou c'est un peu juste », «il ne manque pas grand chose ou cela ne vaut rien ». Et il « chiffre »sa conclusion en fonction de la norme retenue dans son domaine. Qu'on lui dit que réussir exige une cote de $60 \%$ ou de $50 \%$ ne changera rien à sa conclusion. 
Il y aura donc un nivellement par le bas des cotations, mais pas du niveau d'enseignement ! C'est un enseignant qui vous le dit.....

(4b) C'est un enseignant (?) qui vous le dit... cour avec «s » et subjonctif « dise »!!!! C'est le «Bon Usage » qui vous le rappelle...

L'imaginaire des garants classiques de la norme, auquel participe l'enseignant (un bon enseignant ne doit pas commettre de faute), se trouve ici renforcé par le renvoi à une parole grammaticale «d'évangile» : le Bon Usage.

Cependant, les puristes sont aussi pointés du doigt, car pour certains ils seraient assimilables à des trolls déviant une discussion au profit de la disqualification des intervenants et au détriment de l'échange d'arguments sur le sujet même de l'article. C'est la contre-offensive antipuriste qui vise à rééquilibrer la discussion au profit de l'information même, comme l'illustre l'exemple suivant:

(5) PRIÈRE - Un ambassadeur américain à l'ONU incite ses collègues à cesser de négocier ivres [titre] (bigbrowser.blog.lemonde.fr 5.3.2013)

(5a) En l'occurrence, je ne vois pas pourquoi on devrait « évoluer». On traduit généralement « intoxicated » par « ivre », " gris », et intoxiqué par « poisoned ». Les deux mots n’ont pas la même signification, il est inutile d'appauvrir la langue française en détruisant la nuance. Le recours à des mots étrangers n'a d'intérêt que dans la mesure où ils enrichissent la langue. J'ajouterais que les gens qui parlent mal deux langues différentes dans la même phrase me tapent sur les nerfs. On parle la langue que l'on veut, mais on essaye de la parler correctement (c'est à dire sans mettre des citations latines ou des pseudo-anglicismes mal prononcés et mal utilisés à chaque coin de phrase).

(5b) Rholala les lourds! L'info c'est que des lascars en haut lieu negocient et prennent des decisions alors qu'ils sont completement raides. Plus ou moins revoltant en soi. Et bien ici, une poignée de chevaliers defenseurs de la langue francaise, mon cher, se revolte et nous fait un sketch parce que l'auteur a preferé un anglicisme qui fait tres bien l'affaire, l'alcool etant jusqu'a preuve du contraire, toxique, plutot que notre bon vieux mot a nous, qu'on a inventé, et qui nous appartient: ivre... Rendez vous compte mon bon monsieur, il y a plus de respect. Marre de ces lourds qui tous les jours remplissent les colonnes des commentaires avec leur corrections, a croire qu'ils ne lisent l'article que dans l'espoir d'y trouver une horrible faute a denoncer. A la limite, que l'on corrige une faute d'orthographe, soit, une erreur grammaticale, passe encore, mais de la a crier au scandale quand l'auteur use d'un anglicisme tout a fait valable, ca devient plus que casse-c... On n'appauvrit pas la langue francaise en la faisant vivre, c'est pas parce que l'on use d'anglicismes ou d'autres neologismes que les mots bien de chez nous, francais monsieur, vont disparaitre. Par contre les ayatollas de la langue, que vous disparaissiez des blogues, voila qui ne me derangerait guere. (gras des auteures) 
On voit que l'ironie et la violence verbale du ton traduisent une exaspération produite par un comportement qui est donc ressenti comme récurrent et perturbateur: on retrouve également un classique du discours antipuriste, l'exagération dénominative par déplacement de champ topique (« les ayatollas de la langue »), ainsi que le dialogisme humoristique qui singe un discours convenu du puriste chauvin (« rendez-vous compte mon bon monsieur », « les mots bien de chez nous »). Il est à noter cependant que ce type d'intervention, loin de clore le débat sur la langue, cristallise les positions puristes/antipuristes et favorise a contrario la poursuite de l'échange sur le mode métalangagier.

L'exemple (5) révèle également une obsession puriste, une lignée discursive traditionnelle : la condamnation des anglicismes (voir également [6]). Pour les internautes puristes (5a), le journaliste se doit dans la presse francophone de préserver la pureté de la langue. Cette attitude illustre plus que toute autre la figure de l'amoureux de la langue chère aux puristes français, qui la protègent par là du contact linguistique tout en affichant un ethos savant et multilingue.

Le fil de discussion (6) révèle une autre dimension de l'attitude puriste/antipuriste, à savoir la connaissance des usages discursifs sociaux et des routines journalistiques, utilisés ici comme arguments pour ou contre une attitude puriste (23 commentaires sur 79). Les internautes vont ainsi expliciter quelles lois puristes régissent l'écriture sur le journal et/ou un blogue lié au journal : «la qualité grammaticale et orthographique » du célèbre quotidien est mise à mal par la disposition technologique qui mêle et superpose les supports numériques diversifiés:

(6) ÉTRENNES - Le SDF de New York remercie le policier, mais préfère rester pieds nus [titre] (bigbrowser.blog.lemonde.fr 3.12.2012)

- Attention à ne pas traduire systématiquement « police officer » par « officier de police » il s'agit plus probablement ici d'un « agent de police».

- Vous exagérez au Monde : « elle a été likée ».

- Ceci est un blogue, ce n'est donc pas un article du Monde mais un blogue étant hébergé sur la surface numérique que représente le Monde.

- Insupportables ces gens qui bondissent pour rectifier «non non non! c'est un blogue! » On s'en fout ! c'est pareil ! Le Monde est autant responsable des publicités qu'il publie que des blogue qu'il héberge sous son nom, en son nom et de plus sur la première page de son site!

- Oui, mais non : un blogue n'est pas soumis aux secrétariat de rédaction du Monde, responsable de la qualité grammaticale et orthographique. Sinon, oui, ces anglicismes débiles sont vraiment la preuve d'un irrespect total de notre langue. Il ne « préfère » pas mais il craint pour sa vie de porter des chaussures coûteuses. Avec une paire à $20 \$$ il ne marcherait pas pieds nus! Titre racoleur à changer. 
Plusieurs commentaires - (6), (1c), (2a), (3) - présentent une caractéristique distinctive du discours rectificatif des lecteurs : les adresses directes au méta-énonciateur journalistique absent de la situation d'énonciation (Calabrese, 2014a). Même si elles ne sont pas présentes dans chaque énoncé rectificatif, ces interpellations ("Attention à ne pas traduire... », « Vous exagérez au Monde... », «Titre racoleur à changer ») établissent une sorte de proximité avec le discours journalistique qui révèle la place que les publics médiatiques actifs pensent être la leur : celle des surveillants du discours médiatique en général, se plaçant à la même hauteur que le méta-énonciateur. Si l'environnement du blogue est plus propice aux échanges plus équilibrés avec le journaliste (comme on le voit dans les exemples [1b] et [1d]), on constate à la structure des fils de discussion que ce qui prime sont les échanges entre internautes.

L'extrait (7) exemplifie toute la dynamique du purisme dans un contexte numérique. Il est impossible de reproduire tout le fil de discussion (109 commentaires pour l'article en question), aussi nous reprenons les énoncés qui confirment nos observations préliminaires:

(7) CAPRICE - Kim Jong-un mécontent de la pénurie de femmes vierges [titre] (bigbrowser. blog.lemonde.fr 20.8.2013)

- Plusieurs faute d'orthographe et/ou de frappe dans cet article : «cette âge » (au lieu de «cet âge »), « pour la part » au lieu de «pour sa part », mais le plus amusant, c'est d'y évoquer le « trafic d'âtres humains ».

- Je dirais meme plus: « plusieurEs fautes »....

- «plusieurEs »...? vraiment? courrez acheter un Bescherelle!

- CouRRez? Intéressant...

- Ahahaha magnifique échange ! Et vive le Bescherelle et le Petit Larousse !

- L'arroseur arrosé! ha ha!

- Par contre s'interesser un peu au sujet de l'article...

- Ras le bol des dictateurs de l'orthographe, cessez donc d'écrire si la lecture vous insupporte! La modération ferait bien de supprimer vos comms poubelles dont tout le monde s'en fou sauf les vieux schnocks empêtrés dans leur illettrisme compulsif en se sentant obligé de toujours corriger les autres.

- L’orthographe la science des imbéciles...

- Les fautes d'orthographe ne sont pas bien graves par rapport a la médiocrité du monde autrefois journal de référence ........ 
- Habituellement, je ne poste pas de commentaire juste à propos des fautes de frappe et/ou d'orthographe trouvées au sein d'un article de blogue(ue), mais aujourd'hui, il y en avait une (corrigée depuis) qui m’a beaucoup amusé («trafic d'âtres humains » au lieu de « trafic d'êtres humains »). [...] Mais les réactions épidermiques de certains commentateurs sur le sujet plus général de l'orthographe française m'ont depuis presque autant, si ce n'est plus, fait hurler de rire.

Nous y trouvons:

- la déviation de l'objet de discours : 35 commentaires portent directement sur l'orthographe (40 si l'on compte des commentaires indirects). Ils se trouvent majoritairement en début d'article, mais également intercalés avec des commentaires sur le sujet de l'article ;

- les figures de l'arroseur arrosé et du grammar nazi ; l'attitude du puriste bienveillant et celle de l'amoureux de la langue;

- $\quad$ un renvoi à des figures patrimoniales du savoir linguistique ou grammatical, censées représenter la norme (le professeur, le lettré, l'érudit) ;

- le renvoi aux ouvrages emblématiques de la norme (le Bescherelle, le Larousse);

- $\quad$ un rappel de la norme sur un mode d'expert ou un mode ludique ;

- $\quad$ un discours puriste de type performatif (Moschonas, 2008) qui s'énonce sous la forme d'impératifs à caractère correctif : l'abondance de cette modalité illustre une croyance en la dimension pratique du discours puriste.

Ces exemples montrent que la représentation majoritaire de la norme est bâtie sur un imaginaire puriste. Celui-ci, à son tour, s’appuie sur la technologie discursive (dictionnaires, Bescherelle) et le renvoi à des figures emblématiques, ce qui est caractéristique du discours puriste en contexte numérique. Les commentaires portent sur les classiques de l'imaginaire puriste francophone : les anglicismes, la concordance des temps ou l'accord du participe passé, ce qui conforte l'idéologie linguistique dominante depuis plusieurs siècles.

De façon générale, on dira que le discours des internautes censeurs se caractérise par des modalités expressives, fondées sur une émotion (le rapport affectif à la langue) qu'il s'agira de rationaliser par l'appel aux normes et aux règles. À ce sujet, l'un des intervenants qui condamne les interventions des censeurs parle de leurs « réactions épidermiques».

\section{Le purisme en contexte numérique, un nouveau purisme?}

Malgré un corpus réduit, nous pouvons constater que les interventions autour de la langue des journalistes ont des caractéristiques propres dans l'environnement numérique de la Toile. Qu'il contribue à développer un éthos individuel (comme sur Facebook) ou collectif (notamment dans les blogues), le purisme/antipurisme 2.0 possède des caractéristiques propres. En effet, nous consta- 
tons que le commentaire puriste répond à la même logique générale des commentaires sur la Toile: il est compulsif, dans le sens où on épingle la faute ${ }^{3}$ car le dispositif invite l'internaute à se focaliser sur l'écrit et à produire du texte à son tour. Alors que la lettre adressée au journal pour figurer dans le courrier des lecteurs représentait un investissement de temps et d'énergie, et supposait pour cela une motivation intellectuelle suffisamment grande pour justifier l'effort, le journalisme interactif4 non seulement autorise mais encourage l'action de pointer la faute, et développe des routines de lecture chez les lecteurs qui vont dans le sens de la surveillance de l'écriture journalistique (Calabrese 2014b, 2014c). Puisque, dans le contexte de l'Internet, le commentaire puriste a la force de stopper des énoncés et de délégitimer des énonciateurs, il peut être vu comme un outil de contrôle social : réduit à son mésusage, le locuteur « faiseur de faute » est ensuite réduit au silence par la performance double du locuteur « censeur » (Meunier et Rosier, 2012). On voit ici que le discours puriste a une fonction performative (Moschonas, 2008) qui s'exprime particulièrement bien grâce aux modalités injonctives. Il y a donc une illustration pratique de l'influence des internautes dans la construction du savoir langagier.

Dans ce sens, le commentaire métalinguistique sur Internet rencontre la logique du purisme, qui est un outil de contrôle et de sanction dans le domaine de la langue, une doxa normative. Mais il a également une logique propre, il est symptomatique des pratiques numériques, absolument ancrées dans la lecture et l'écriture autant que dans le dialogue. Dans ce sens, la Toile (et la forme d'énoncé qu'elle a tendance à produire : le commentaire) est un environnement privilégié pour la gestation et la circulation d'attitudes puristes, et présente une différence significative avec le purisme pré-Internet, qui se déployait soit sur un terrain écrit (et donc une communication asynchrone), soit dans la conversation (échange synchrone). Autrement dit, la conjonction de l'écrit et du dialogue encourage les attitudes puristes et leurs contre-discours, l'antipurisme. Cet environnement particulier permet de se focaliser sur l'écrit tout en s'adressant au fauteur, qu'il soit journaliste ou internaute, ce qui permet à l'argument puriste (ou antipuriste) de devenir un argument ad hominem qui délégitime l'énonciateur, ou bien de confirmer le rôle des publics dans le dispositif médiatique, à savoir celui des gardiens des normes, qu'elles soient linguistiques ou ayant trait aux routines scripturaires des journalistes.

Mais ce discours permet également de créer des communautés de valeurs en divisant le lectorat entre puristes et antipuristes. Dans le blogue, on observe que les commentaires portant sur la langue sont surreprésentés - 23/79 pour l'exemple (6), 35/109 pour l'exemple (7) - et qu'ils démarrent des fils de discussion très longs, ce qui signifie qu'il y a une déviation plus importante de l'objet de discours mais aussi plus de place pour l'échange entre internautes. Le rapport que ceux-ci entretiennent avec l'auteur est également plus proche qu'avec d'autres journalistes, de même que celui qu'ils entre-

3. «Faute » recouvre ici à la fois une erreur orthographique et/ou grammaticale réelle (une lettre qui manque, un accord non fait), mais aussi dans la pratique des internautes des cas qui ne sont pas strictement des manquements à des règles de grammaire mais plutôt des variations non acceptées par les puristes de la Toile (par exemple le manquement à la concordance des temps).

4. Celui qui permet aux usagers d'interagir entre eux et éventuellement avec les journalistes (Nip, 2006). 
tiennent avec les autres commentateurs, au point de créer de véritables communautés discursives. La question des communautés devient ici cruciale car elle serait liée au dispositif énonciatif propre du blogue, mais également au contrat de communication qui s'établit avec les lecteurs, et qui ne serait pas le même dans un blogue de journaliste, même s'il est incorporé au journal et qu'il produit des articles d'information.

\section{Conclusion}

Comment le purisme participe-t-il de la construction des idéologies discursives en contexte journalistique? Le dispositif des commentaires fait émerger des représentations sur le journalisme, les journalistes (ou plus globalement sur l'énonciation éditoriale, comme l'appellent Jeanneret et Souchier [2005]) et le discours d'information qui révèlent le rôle que les publics médiatiques pensent être celui de la presse (Calabrese, 2014b). Deux grandes postures d'internautes se dégagent : le puriste et l'antipuriste; si le dernier se veut un stoppeur du discours normatif, il ne fait au contraire que le stimuler (comme le premier d'ailleurs). Dans le contexte du dispositif d'intervention numérique, la construction de deux «camps» (avec son lot de dénominations pour les uns et les autres, ses injonctions et ses prédiscours plus ou moins figés) incite au débat, et même à la constitution de communautés virtuelles comme c'est le cas des blogues, où les mêmes pseudonymes reviennent et les échanges ressemblent bien plus à un polylogue que dans la section des commentaires des journaux. Les deux catégories d'internautes font preuve d'une connaissance de l'environnement numérique, par le biais d'énoncés qui jugent de la pertinence ou non des commentaires sur la correction de la langue. Ainsi, on peut dire que les postures normative et antinormative se construisent non seulement sur un savoir savant sur la langue, mais sur des prédiscours liés au rôle de la presse écrite et sur une connaissance empirique des genres médiatiques. Les deux postures se construisent ainsi sur une énonciation experte.

C'est particulièrement dans les blogues, c'est-à-dire là où des communautés virtuelles sont plus à même de se constituer, que la correction linguistique entraine une déviation de l'objet de discours, puisque les internautes vont plus facilement s'engager dans une conversation et donc suivre des fils de discussion, alors que dans le journal à proprement parler il y aura moins d'interaction. C'est la dynamique de l'échange qui va permettre aux lecteurs de blogue d'adopter un ethos à la fois savant et ludique, que l'on retrouve moins dans les commentaires sur l'espace du journal, beaucoup plus axiologiques et laconiques, la dimension interactive entre les internautes étant moins présente. Cette dimension interactive permet de montrer les différentes formes d'échange en contexte numérique et la manière dont la norme (ou son contraire) se construit. Dans le blogue, on s'adresse plus souvent à ses pairs qu'au journal, ce qui montre que l'orthographe et l'écriture journalistique sont un excellent déclencheur de conversation (on le voit dans la structure des échanges). Au contraire, dans le contexte plus « institutionnel » du journal, les internautes auront tendance à produire des énoncés d'adresse à l'énonciateur journalistique, très normatifs et avec peu de place pour la négociation (comme nous l'avons exposé dans Calabrese [2014b]), ce qui montre à la fois une représentation des 
journalistes par rapport à la langue et le rôle de chaque dispositif sociotechnique sur la Toile. Cette posture conforte l'image de la presse écrite comme gardienne de la pureté linguistique, et du public comme une entité de contrôle para-institutionnelle. 


\section{Références}

Amossy, Ruth (2011), «La coexistence dans le dissensus : la polémique dans les forums de discussion », Semen, n³1, p. 25-42.

Bruns, Alex (2010), « News Produsage in a Pro-Am Mediasphere », dans Graham Meikle et Guy Redden (dir.), News Online : Transformations and Continuities, London, Palgrave Macmillan.

Calabrese Laura (dir.) (2011), L'Internet, corpus sauvage : nouvelles ressources, nouveaux problèmes, numéro thématique de la revue Le discours et la langue, $n^{\circ} 2.1$.

Calabrese, Laura (2014a), « Rectifier le discours d'information médiatique : quelle légitimité pour le discours profane dans la presse d'information en ligne? », Carnets du Cédiscor, nº 12, p. 21-34.

Calabrese, Laura (2014b), « Le discours prescriptif des internautes sur la presse d'information généraliste », communication au colloque Reprises et métamorphoses de l'actualité : fabrication, légitimation, et représentations de l'information, Lyon, 27-28 mars 2014.

Calabrese, Laura (2014c), «Paroles de lecteurs : un objet de recherche hybride en sciences du langage », Studii de lingvistică, $n^{\circ} 4$ (Écritures et genres numériques, sous la dir. de Aleksandra Nowakowska et François Perea), p. 13-27.

Canut, Cécile (2007), « L'épilinguistique en question », dans Gilles Siouffi et Agnès Steuckardt (dir.), Les linguistes et la norme : aspects normatifs du discours linguistique, Berne, Peter Lang, p. 49-72.

Hild, Mat (2012), Et toi, t'es qui ? Petite typologie des profils facebook, Paris, Flammarion.

Jeanneret, Yves et Emmanuël Souchier (2005), « L'énonciation éditoriale dans les écrits d'écran », Communication et langages, n 145, p. 3-15.

Meunier, Deborah et Laurence Rosier (2012), « La langue qui fâche : quand la norme qui lâche suscite l'insulte », Argumentation et analyse du discours, n’ 8, disponible sur http://aad.revues.org/1285. [Page consultée le 26 mars 2014.]

Meunier, Deborah et Laurence Rosier (2014), «L La recette commentée : un genre de discours renouvelé ?», Arena Romanistica, n²13, p. 224-243.

Moschonas, Spiros (2008), «Vers une théorie performative du purisme », Le Français Moderne, n 76 (Nouveaux regards sur le purisme, sous la dir. de Laurence Rosier), p 38-50.

Nip, Joyce (2006), «Exploring the Second Phase of Public Journalism », Journalism Studies, vol. 7, $n^{\circ} 2$, p. 212-236.

Osthus, Dietmar (2004), « Le bon usage d'Internet : le discours normatif sur la Toile », disponible sur http://www.dietmar-osthus.de/norme.htm. [Page consultée le 26 mars 2014.]

Paveau, Marie-Anne et Laurence Rosier (2008), La langue française : passions et polémiques, Paris, Vuibert. 
Rosier, Laurence (dir.) (2008), Nouveaux regards sur le purisme, numéro thématique de la revue Le Français moderne, $n^{\circ} 71$.

Souchier, Emmanuël (1996), «L'écrit d'écran, pratiques d'écriture informatique », Communication et langages, $n^{\circ} 107$, p. 105-119. 